PROCEEDINGS OF THE

AMERICAN MATHEMATICAL SOCIETY

Volume 127, Number 10, Pages 2865-2869

S 0002-9939(99)04827-3

Article electronically published on April 23, 1999

\title{
SINGULAR MEASURES WITH ABSOLUTELY CONTINUOUS CONVOLUTION SQUARES ON LOCALLY COMPACT GROUPS
}

\author{
ANTONIS BISBAS
}

(Communicated by Christopher D. Sogge)

\begin{abstract}
Saeki's result states that on any locally compact nondiscrete group there exist continuous singular measures, with respect to the left Haar measure, $\mu$ with $\mu * \mu$ in $L^{p}$ for all $p, 1 \leq p<\infty$. This paper gives a new and short proof of this using Rademacher-Riesz products.
\end{abstract}

\section{INTRODUCTION}

In 1938, Wiener and Wintner [9], [3], p.209, proved that there exists a singular probability measure $\mu$ on $[-\pi, \pi]$ such that $\mu * \mu$ is absolutely continuous. In 1966, Hewitt and Zuckerman [4] showed that every nondiscrete locally compact abelian group supports such a measure (absolutely continuous with respect to Haar measure) and the Lebesgue-Radon-Nikodým derivative of $\mu * \mu$ is in $L^{p}$ for all $p, 1 \leq p<\infty$. Hewitt has asked whether such measures exist on every (locally) compact group [7], p.217. Ragozin [7] proved that every connected compact simple Lie group has a measure with absolutely continuous square. In 1977 Saeki [8], p. 403, proved Hewitt's question. We also note two papers on this subject. One by Karanikas and Koumandos [6] which makes use of the Rademacher-Riesz products, and another by Dooley and Gupta [2] which makes use of the theory of compact Lie groups. In this paper we give a new and short proof of Saeki's result. Our ideas have been adapted from [6]; see also [1] and [5]. Section 2 contains a brief summary, for the reader's convenience, of some results and techniques from [6] that we shall need to use. Our proof of the main theorem appears in section 3.

\section{Terminology}

Let $G$ be a locally compact, nondiscrete metrizable group and $E$ a compact subset of $G$ with left Haar measure equal to one. We define a system of Rademacher functions $\left(r_{n}\right)_{n=1}^{\infty}$ as follows: We divide $E$ into two disjoint subsets, $E_{11}, E_{12}$ of equal measure and define $r_{1}(x)=1$ for $x \in E_{11}$ and $r_{1}(x)=-1$ for $x \in E_{12}$. Similarly, we divide each of these two subsets $E_{11}, E_{12}$ to define $r_{2}$, etc. We define the Walsh functions $\left(w_{n}\right)_{n=0}^{\infty}$ by the relations:

$$
w_{n}=r_{j_{1}} r_{j_{2}} \ldots r_{j_{p}}, \quad n=2^{j_{1}-1}+2^{j_{2}-1}+\ldots+2^{j_{p}-1}, \quad j_{1}<j_{2} \ldots<j_{p} .
$$

Received by the editors June 25, 1997 and, in revised form, December 3, 1997.

1991 Mathematics Subject Classification. Primary 43A05.

Key words and phrases. Singular measures, Rademacher functions.

(C)1999 American Mathematical Society 
The existence is given by the following Lemma:

Lemma 1. On any nondiscrete metrizable group we can find a set $E$ and a partition into sets of equal measure, $E_{n, k}, 1 \leq k \leq 2^{n}$, such that:

(i) $\max _{1 \leq k \leq 2^{n}} \operatorname{diam} E_{n, k} \rightarrow 0$, as $n \rightarrow 0$, (diam $E_{n, k}$ denotes the diameter of $E_{n, k}$ with respect to the metric of $G$ ).

(ii) The Walsh system is complete in $L^{2}(E)$.

(iii) If $f, g \in L^{1}(G)$, then for any $p \in[1, \infty), \lim _{n \rightarrow \infty} \int f w_{n}=0, \lim _{n \rightarrow \infty}\left\|f *\left(g w_{n}\right)\right\|_{p}=$ 0 and $\lim _{n \rightarrow \infty}\left\|\left(g w_{n}\right) * f\right\|_{p}=0$.

Proof. For (i) and (ii) see [6], Lemma 2.1, and for (iii) see the proof of Lemma 3.2 in $[6]$.

We have also (see [1], [5], [6]) that

Lemma 2. Let $f_{n}(x)=\prod_{j=1}^{n}\left(\chi_{E}(x)+a_{j} r_{j}(x)\right)$, where $\chi_{E}$ is the characteristic function of $E$ and $\left|a_{j}\right| \leq 1$. Then

(i) The sequence $f_{n}$ converges weak $k^{*}$ to some $\mu$ in $M(G)$, the convolution algebra of bounded complex-valued Borel measures on $G$.

(ii) The measure $\mu$ is singular if and only if $\sum_{n=1}^{\infty} a_{n}^{2}=\infty$.

(iii) The measure $\mu$ is continuous if and only if $\sum_{n=1}^{\infty}\left(1-\left|a_{n}\right|\right)=\infty$.

\section{The MAin THEOREM}

Theorem 1. Let $G$ be a nondiscrete locally compact metrizable group. Then there exists a continuous singular measure $\mu \in M(G)$ with absolutely continuous convolution square and the Lebesgue-Radon-Nikodým derivative of $\mu * \mu$ is in $L^{p}(G)$ for all $p, 1 \leq p<\infty$.

Proof. Let $\left(a_{n}\right)_{n=1}^{\infty}$ be a sequence such that $\sum_{n=1}^{\infty} a_{n}^{2}=\infty, \quad \sum_{n=1}^{\infty} a_{n+1}^{4}\left(\sum_{k=1}^{n} a_{k}^{2}\right)^{q}<\infty$, for any $q \in \mathbb{N}$ and $\left|a_{n}\right| \leq 1$. We shall show that there exists a subsequence $\left(r_{m_{k}}\right)_{k=1}^{\infty}$ of Rademacher functions such that if $f_{n}(x)=\prod_{k=1}^{n}\left(\chi_{E}(x)+a_{k} r_{m_{k}}(x)\right)$, then the weak* limit $\mu$ of $f_{n}$ is a continuous singular measure $\mu \in M(G)$, with absolutely continuous convolution square and the Lebesgue-Radon-Nikodým derivative of $\mu * \mu$ is in $L^{p}(G)$ for all $p, 1 \leq p<\infty$. From Lemma 2 we have that $\mu$ is a continuous singular measure. It is easy to show that $f_{n} * f_{n} \rightarrow \mu * \mu, n \rightarrow \infty$, weak* in $M(G)$ and so from completeness of $L^{p}$ it is enough to show that $f_{n} * f_{n}$ is a Cauchy sequence in $L^{p}$. It is also enough to prove this only for $p$ an even integer. For simplicity of notation we write

$$
\begin{gathered}
t_{n}=f_{n} * f_{n}, g_{n}=\left(f_{n} r_{m_{n+1}}\right) * f_{n}, h_{n}=f_{n} *\left(f_{n} r_{m_{n+1}}\right), \\
s_{n}=\left(f_{n} r_{m_{n+1}}\right) *\left(f_{n} r_{m_{n+1}}\right), n \in \mathbb{N} .
\end{gathered}
$$

We shall estimate the norm $\left\|t_{n}-t_{m}\right\|_{p}, n>m$. Schwarz's inequality yields

$$
\left\|t_{n}-t_{m}\right\|_{p}^{p}=\left\|\sum_{i=m}^{n-1}\left(t_{i+1}-t_{i}\right)\right\|_{p}^{p}=\left\|\sum_{i=m}^{n-1}\left[a_{i+1}\left(g_{i}+h_{i}\right)+a_{i+1}^{2} s_{i}\right]\right\|_{p}^{p}
$$




$$
\begin{gathered}
=\sum_{\nu=0}^{p}\left(\begin{array}{l}
p \\
\nu
\end{array}\right) \int\left[\sum_{i=m}^{n-1} a_{i+1}\left(g_{i}+h_{i}\right)\right]^{p-\nu}\left[\sum_{i=m}^{n-1} a_{i+1}^{2} s_{i}\right]^{\nu} \\
\leq \sum_{\nu=0}^{p}\left(\begin{array}{l}
p \\
\nu
\end{array}\right)\left(\int\left[\sum_{i=m}^{n-1} a_{i+1}\left(g_{i}+h_{i}\right)\right]^{2(p-\nu)}\right)^{\frac{1}{2}}\left(\int\left[\sum_{i=m}^{n-1} a_{i+1}^{2} s_{i}\right]^{2 \nu}\right)^{\frac{1}{2}} .
\end{gathered}
$$

For $\nu \geq 1$ we have

$$
\begin{aligned}
\int\left[\sum_{i=m}^{n-1} a_{i+1}^{2} s_{i}\right]^{2 \nu}= & \sum_{i=m}^{n-1} a_{i+1}^{4 \nu} \int s_{i}^{2 \nu} \\
& +\sum_{k=m+1}^{n-1} \sum_{l=1}^{2 \nu-1}\left(\begin{array}{c}
2 \nu \\
l
\end{array}\right) \int\left[\left(a_{k+1}^{2} s_{k}\right)^{l}\left(\sum_{i=m}^{k-1} a_{i+1}^{2} s_{i}\right)^{2 \nu-l}\right] .
\end{aligned}
$$

Using Lemma 1(iii) and Fubini's theorem we find $\left(r_{m_{k}}\right)_{k>1}$ such that

$$
\max \left\{\left|\int\left[s_{k}\left(\sum_{i=m}^{k-1} a_{i+1}^{2} s_{i}\right)^{2 \nu-1}\right]\right|, \quad 1 \leq \nu \leq k, \quad 1 \leq m \leq k-1\right\} \leq \frac{1}{k^{2}}
$$

and, at the same time, using Lemma 1(iii),

$$
\max \left\{\left\|g_{i}\right\|_{k}, \quad\left\|h_{i}\right\|_{k}, \quad k=1, \ldots, 2 i\right\}<\frac{1}{2 i^{3}}, \quad i=1,2 \ldots .
$$

Thus, for $p<m<n$, using Minkowski's inequality we obtain

$$
\begin{gathered}
\left\|t_{n}-t_{m}\right\|_{p}^{p} \leq \frac{1}{m^{2 p}}+\sum_{\nu=1}^{p}\left(\begin{array}{l}
p \\
\nu
\end{array}\right) \frac{1}{m^{2(p-\nu)}}\left[\sum_{i=m}^{n-1} a_{i+1}^{4 \nu} \int s_{i}^{2 \nu}+2 \nu \sum_{k=m+1}^{n-1} \frac{1}{k^{2}}\right. \\
\left.+\sum_{k=m+1}^{n-1} \sum_{l=2}^{2 \nu-1}\left(\begin{array}{c}
2 \nu \\
l
\end{array}\right) \int\left[\left(a_{k+1}^{2} s_{k}\right)^{l}\left(\sum_{i=m}^{k-1} a_{i+1}^{2} s_{i}\right)^{2 \nu-l}\right]\right]^{\frac{1}{2}} .
\end{gathered}
$$

Applying Schwarz's and Minkowski's inequalities successively, in the last term of the above, we observe that, in view of our hypothesis on the sequence $\left(a_{n}\right)_{n=1}^{\infty}$ and since

$$
\left|s_{n}\right| \leq\left|t_{n}\right|, \quad n \in \mathbb{N}
$$

it suffices to prove that $\sup _{n}\left\|t_{n}\right\|_{p}<c_{p}$, where $c_{p}$ is a constant which depends only on $p$. We have

$$
\begin{aligned}
\left\|t_{n+1}\right\|_{p}^{p} & =\left\|t_{n}+a_{n+1}\left(g_{n}+h_{n}\right)+a_{n+1}^{2} s_{n}\right\|_{p}^{p} \\
& =\sum_{k_{2}=0}^{p} \sum_{k_{1}=0}^{k_{2}}\left(\begin{array}{c}
p \\
k_{2}
\end{array}\right)\left(\begin{array}{c}
k_{2} \\
k_{1}
\end{array}\right) C_{p, n}\left(k_{1}, k_{2}\right),
\end{aligned}
$$

where

$$
C_{p, n}\left(k_{1}, k_{2}\right)=a_{n+1}^{2 p-k_{2}-k_{1}} \int t_{n}^{k_{1}}\left(g_{n}+h_{n}\right)^{k_{2}-k_{1}} s_{n}^{p-k_{2}} .
$$

If $k_{1}<k_{2}$, then by Lemma 1 (iii) and (3) we find $r_{m_{n+1}}$ such that

$$
\max \left\{\left|C_{p, n}\left(k_{1}, k_{2}\right)\right|, \quad 0 \leq k_{1}<k_{2} \leq p, \quad 2 \leq p \leq n+1\right\} \leq \frac{a_{n+1}^{4}}{3^{n+1}} \min _{2 \leq k \leq n+1}\left\|t_{n}\right\|_{k}^{k} .
$$


If $k_{1}=k_{2}$, then we have

$$
C_{p, n}\left(k_{1}, k_{1}\right)=a_{n+1}^{2\left(p-k_{1}\right)} \int t_{n}^{k_{1}} s_{n}^{p-k_{1}} .
$$

If $k_{1} \leq p-2$, then using (3) we have

$$
\begin{aligned}
\sum_{k_{1}=0}^{p-2}\left(\begin{array}{c}
p \\
k_{1}
\end{array}\right)\left|C_{p, n}\left(k_{1}, k_{1}\right)\right| & \leq \sum_{k_{1}=0}^{p-2}\left(\begin{array}{c}
p \\
k_{1}
\end{array}\right) a_{n+1}^{2\left(p-k_{1}\right)}\left|\int t_{n}^{k_{1}} g_{n}^{p-k_{1}}\right| \\
& \leq a_{n+1}^{4}\left(2^{p}-(p+1)\right)\left\|t_{n}\right\|_{p}^{p} .
\end{aligned}
$$

If $k_{1}=p-1$, then Fubini's theorem and Lemma 1(iii) show that we can find $r_{m_{n+1}}$ such that

$$
\max _{2 \leq p \leq n+1}\left|C_{p, n}(p-1, p-1)\right| \leq a_{n+1}^{4} \min _{2 \leq k \leq n+1}\left\|t_{n}\right\|_{k}^{k}
$$

Finally, for $k_{1}=p$ we have

$$
\left|C_{p, n}(p, p)\right| \leq\left\|t_{n}\right\|_{p}^{p}
$$

Subsequently, we can find $r_{m_{n+1}}$ satisfying (4) and (5) such that

$$
\left\|t_{n+1}\right\|_{p}^{p} \leq\left(1+2^{p} a_{n+1}^{4}\right)\left\|t_{n}\right\|_{p}^{p}, \quad 2 \leq p \leq n+1 .
$$

This implies that $\sup _{n}\left\|t_{n}\right\|_{p}<c_{p}$. It is clear that we can find a subsequence $\left(r_{m_{k}}\right)_{k=1}^{\infty}$, using induction, satisfying the relations (1), (2), (4) and (5) simultaneously. This completes the proof.

Corollary 1. The result of Theorem 1 holds for any nondiscrete locally compact group.

Proof. As in [6]; see also [10].

\section{ACKNOWLEDGEMENT}

The author wishes to thank C. Karanikas for some helpful comments and suggestions.

\section{REFERENCES}

[1] A. Bisbas and C. Karanikas, On the continuity of measures, Applicable Analysis, Vol 48 (1993), 23-35. MR 95e:43001

[2] A. H. Dooley and S. K. Gupta, Continuous singular measures with absolutely continuous convolution squares, Proceedings of the American Math. Soc. Vol. 124, 10, (1996), 3115 3122. MR 96m: 43011

[3] C. C. Graham and O. C. McGehee, Essays in Commutative Harmonic Analysis, SpringerVerlag, New York 1979. MR 81d:43001

[4] E. Hewitt and H. Zuckerman, Singular measures with absolutely continuous convolution squares, Proc. Cambridge Phil. Soc. 62 (1966), 339-420; 63, (1967), 367-368. MR 33:1655

[5] C. Karanikas, Examples of Riesz products-type measures on metrizable groups, Boll. Un. Math. Ital. 7, 4-A (1990), 331-341. MR 92d:43002

[6] C. Karanikas and S. Koumandos, Continuous singular measures with absolutely continuous convolution squares on locally compact groups, Illinois J. of Math. 35 (3) (1991), 490-495. MR 92b:43004

[7] D. L. Ragozin, Central measures on compact simple Lie groups, J. of Functional Analysis 10 (1972), 212-229. MR 49:5715

[8] S. Saeki, Singular measures having absolutely continuous convolution powers, Illinois J. of Math. 21 (1977), 395-412. MR 58:6719 
[9] N. Wiener and A. Wintner, Fourier-Stieltjes transforms and singular infinite convolutions, Amer. J. Math., vol. 60 (1938), 513-522.

[10] J. H. Williamson, A theorem on algebras of measures on topological groups, Proc. Edinburgh Math. Soc., vol. 11 (1958/59), 195 - 206. MR 22:2851

Department of Mathematics, University of the Aegean, Karlovasi 83200, Samos, GREECE

Current address: Technological Education Institute of Kozani, School of Technological Applications, General Department, Kila 50100, Kozani, Greece

E-mail address: bisbas@kozani.teikoz.gr 